\title{
BAO plate archive project: digitization, electronic database and scientific usage
}

\author{
A. M. Mickaelian, K. S. Gigoyan, G. A. Mikayelyan, G. M. Paronyan, M. V. Gyulzadyan, and \\ G. R. Kostandyan
}

NAS RA V. Ambartsumian Byurakan Astrophysical Observatory (BAO), Byurakan 0213, Aragatzotn province, Armenia

\begin{abstract}
Observational archives are the main source for most of the astronomical studies, as given observations cannot entirely solve any problem and need to be complemented by related observing data both for construction of the time domain, multiwavelength, multi-technique and multi-method picture. This relates to both modern observations and historical ones preserved at many observatories. Therefore, digitization and accessibility of all possible data are rather important. The Byurakan Astrophysical Observatory (BAO) Plate Archive consists of some 37,500 photographic plates and films, obtained with $2.6 \mathrm{~m}$ telescope, $1 \mathrm{~m}$ and $0.5 \mathrm{~m}$ Schmidt telescopes and other smaller ones during 1947-1991 and then by digital methods since 1996. Its most important part, the famous Markarian Survey (or the First Byurakan Survey, FBS) 1874 plates were digitized in 2002-2007 and the Digitized FBS (DFBS, www.aras.am/Dfbs/dfbs.html) was created. New science projects have been conducted based on this low-dispersion spectroscopic material. Several other smaller digitization projects have been carried out as well, such as part of the Second Byurakan Survey (SBS) plates, photographic chain plates in Coma, where the blazar ON 231 is located and $2.6 \mathrm{~m}$ film spectra of FBS Blue Stellar Objects. However, most of the plates and films were not digitized. In 2015, we have started a project on the whole BAO Plate Archive digitization and its scientific usage. It was aimed at digitization, extraction and analysis of archival data and building an electronic database and interactive sky map. Armenian Virtual Observatory (ArVO, www.aras.am/Arvo/arvo.htm) database will accommodate all new data. The project runs in collaboration with the Armenian Institute of Informatics and Automation Problems (IIAP) and continued during 4 years in 2015-2018. Later on, the project was renovated for 2020-2021. The final result will be an Electronic Database and online Interactive Sky map to be used for further research projects. ArVO will provide all standards and tools for efficient usage of the scientific output and its integration in international databases.
\end{abstract}

Keywords: photographic plates - photographic films - digitization - astrometry - photometry - spectroscopy - astronomical archives - databases - virtual observatories

\section{Introduction}

The astronomical archives created on the basis of numerous observations at many observatories are the most important part of both astronomical observational heritage (plate and film archives) and modern observations (modern archives). The necessity of digitization of astronomical plates was emphasized and current progress in various national and international projects was given at Astroplate workshops (e. g. Osborn (2014); Hudec (2014); Kazantseva (2014); Nesci et al. (2014a); Stupka \& Benesova (2014)).

The Byurakan Astrophysical Observatory (BAO) Plate Archive is one of the largest astronomical archives in the world and is considered to be BAO main observational treasure. It is the results of decades' hard work of Armenian astronomers and the work of BAO telescopes and other expensive equipment, as well as the results of their activities. Today BAO archive holds some 37,500

*aregmick@yahoo.com, Corresponding author 
astronomical plates, films or other carriers of observational data. However, previous observational and informational registration methods currently do not make it available to wide range of scientists, and especially its usage for solution of new research problems. Digitization of BAO plates is a significant contribution to the Wide-Field Plate DataBase (WFPDB) developed in Sofia, Bulgaria (Tsvetkov \& Tsvetkova, 2012).

A project on Digitization of BAO Plate Archive and creation of BAO Interactive Astronomical Database (shortly BAO Plate Archive project, BAO PAP) started in February 2015. It was aimed at preservation of BAO valuable observational material accumulated during 1947-1991, creation of full Database of all BAO observations, creation of BAO Interactive Sky Map with visualization of all observations and quick access to the data, development and accomplishment of new research projects based on the existing observational material, and integration of BAO observations into the international databases. A number of BAO young astronomers were involved in this project and it lasted 4 years. Later on, the project was renovated for 2020-2021 to complete the full tasks.

The project objectives are the preservation of BAO observational archive, preservation of scientific information contained in photographic plates and other careers, creation of opportunity of dissemination and wide usage of observational data, putting in correspondence of observational material to modern standards and usage methods, proposing new science projects and creation of possibility of their further accomplishment, and making BAO activities visible.

A short description of BAO Plate Archive was given by Mickaelian (2014a) and more detailed paper is given in Mickaelian et al. (2016b).

\section{BAO telescopes and observing programmes}

BAO observers worked with a number of BAO telescopes during 1947-1991 and obtained several dozens of thousands of plates, films and other products. The table gives general understanding on observations of $10 \mathrm{BAO}$ telescopes that worked on photographic photometry, electrophotometry, slit and objective prism spectroscopy, and polarimetry of many thousands astronomical objects.

Table 1. Overview of BAO telescopes and produced observational material.

\begin{tabular}{|l|c|c|l|r|}
\hline Telescope & Sizes $(\mathrm{cm})$ & Years & Observing methods & Plates \\
\hline 5" double-astrograph & 13 & $1947-1950$ & photometry & 3000 \\
\hline 6" & 15 & $1947-1950$ & photometry & 3000 \\
\hline 8" Schmidt & $20 / 20 / 31$ & $1949-1968$ & photometry & 4500 \\
\hline 20" Cassegrain & $51 / 800$ & $1952-1991$ & electrophotometry & \\
\hline 10" telescope-spectrograph & 25 & $1953-19 ? ?$ & spectra & \\
\hline Nebular spectrograph & & $1954-19 ? ?$ & spectra & \\
\hline 16" Cassegrain & $41 / 400$ & $1955-1991$ & electrophotometry & \\
\hline 21" Schmidt & $53 / 53 / 183$ & $1955-1991$ & photometry & 12000 \\
\hline 40" Schmidt (AZT-10) & $102 / 132 / 213$ & $1960-1991$ & photometry, spectra & 7500 \\
\hline ZTA-2.6m & $264 / 1016$ & $1975-1991$ & photometry, spectra & 7000 \\
\hline All telescopes & & $\mathbf{1 9 4 7 - 1 9 9 1}$ & & $\mathbf{3 7 0 0 0}$ \\
\hline
\end{tabular}

We give in Table 1 an overview of BAO telescopes and produced observational material. Telescope "Sizes" are given for the mirror and focal length for classical telescopes and for the correcting lens, mirror and focal length for Schmidt type telescopes. Here we list the main observational projects accomplished on the three most important BAO telescopes $(2.6 \mathrm{~m}$ classical reflector, $1 \mathrm{~m}$ Schmidt and 0.5m Schmidt; Figure 1).

\section{1" (0.5m) Schmidt telescope:}

- Polarization of cometary nebula NGC 2261

- Nuclei of nearby Sa and Sb galaxies 


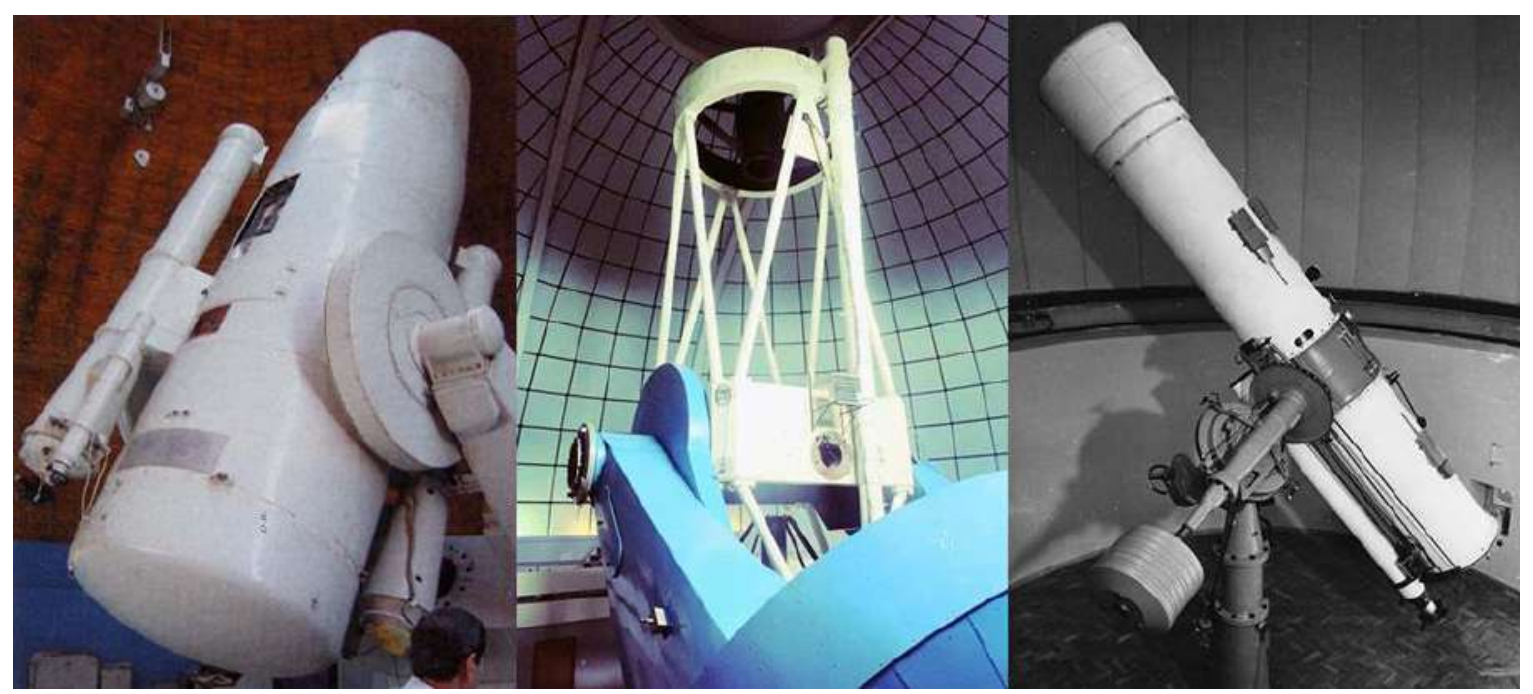

Figure 1. BAO most important telescopes (from left to right): $1 \mathrm{~m}$ Schmidt, $2.6 \mathrm{~m}$ classical reflector and $0.5 \mathrm{~m}$ Schmidt.

- Nuclei of nearby Sc galaxies

- Search for flare stars in Pleiades

- Search for flare stars in Orion

- Search for flare stars in NGC 7000 (Cygnus)

- Search for flare stars in Praesepe

- Search for flare stars in Taurus Dark Clouds (TDC)

- Variability of Markarian galaxies

- Monitoring of extragalactic supernovae in certain areas

\section{0" (1m) Schmidt telescope:}

- Detailed colorimetry of bright galaxies

- First Byurakan Survey (FBS, Markarian survey; Markarian et al. (1989))

- Search for flare stars in Pleiades

- Search for flare stars in Orion

- Search for flare stars in NGC 7000 (Cygnus)

- Search for flare stars in Praesepe

- Search for flare stars in Taurus Dark Clouds (TDC)

- Second Byurakan Survey (SBS; Stepanian (2005))

- Extension of the FBS in the Galactic Plane 


\section{ZTA-2.6m telescope:}

- Morphological study of Markarian galaxies

- Investigation of star clusters

- Investigation of groups and clusters of galaxies

- Spectroscopy FBS blue stellar objects

- Spectroscopy FBS late-type stars

- Spectroscopy SBS galaxies and stellar objects (BAO/SAO)

- Direct images of the central regions of Markarian galaxies

- Spectroscopy of T Tauri and flare stars

- Spectroscopy of Byurakan-IRAS Galaxies (BIG objects)

- Spectroscopy of ROSAT AGN candidates (BAO/HS/OHP/INAOE)

Summarizing, the main observational projects run on these telescopes were:

21" (0.5m) Schmidt: Polarization of cometary nebula NGC 2261, Nuclei of nearby Sa and Sb galaxies, Nuclei of nearby Sc galaxies, Search for flare stars in Pleiades, Orion, NGC 7000 (Cygnus), Praesepe and Taurus Dark Clouds (TDC), Variability of Markarian galaxies, Monitoring of extragalactic supernovae in certain areas, etc.;

40" (1m) Schmidt: the First Byurakan Survey (FBS, Markarian survey; Markarian et al. (1989)), the Second Byurakan Survey (SBS; Stepanian (2005)), Extension of the FBS in the Galactic Plane, Detailed colorimetry of bright galaxies, Search for flare stars in Pleiades, Orion, NGC 7000 (Cygnus), Praesepe and Taurus Dark Clouds (TDC), etc.;

ZTA-2.6m telescope: Morphological study of Markarian galaxies, Investigation of star clusters, Investigation of groups and clusters of galaxies, Spectroscopy of FBS blue stellar objects, FBS late-type stars, SBS galaxies and stellar objects (BAO/SAO), T Tauri and flare stars, Byurakan-IRAS Galaxies (BIG objects) and ROSAT AGN candidates (BAO/HS/OHP/INAOE), and Direct images of the central regions of Markarian galaxies.

Especially efficient were Byurakan spectroscopic surveys accomplished by Markarian and colleagues with $1 \mathrm{~m}$ Schmidt telescope: FBS and SBS.

\section{BAO Plate Archive Project}

The digitization of astronomical plates and films pursues not only the maintenance task, but also it will serve as a source for new scientific research and discoveries, if only the digitized material runs according to modern standards and, due to its accessibility, it will become an active archive. The project is aimed at compilation, accounting, digitization of $\mathrm{BAO}$ observational archive photographic plates and films, as well as their incorporation in databases with modern standards and methods, providing access for all observational material and development of new scientific programs based on this material.

Scientific Programs Board (SPB) was created to evaluate the existing observational material, to select sets of priorities to be scanned first and to propose new research projects. It consists of BAO most experienced observers, as well as researchers from NAS RA Institute of Informatics and Automation Problems (IIAP) are involved for their experience in computer science related to databases and computational methods. Project Executing Team (PET) consists of more than 10 members led by the Head of BAO Astroinformatics Department Gor Mikayelyan, and the members are involved in scanning and reduction of data.

The project consists of the following tasks: 
- Development of technical principles of the Project, necessary Equipment, Timeline and the Budget

- Collection of all photographic plates (until recently only plates obtained before 1974 had been collected in BAO Plate Archive)

- Revision and accounting of the plates and observing journals in BAO Plate Archive,

- Scanning of a few dozens of plates for test and educational reasons to set up the necessary parameters for the scanning in frame of the main Project

- Input of data from observing journals; Creation of the Project Database and development of the principles of organization of data in it

- Creation of the Project Webpage and User Interface

- Scanning of photographic plates and films

- Astrometric solution; Extraction of images and spectra; Wavelength calibration; Density and flux calibration; Multiband (UBVR) photometry

- Making up template low-dispersion spectra; Numerical classification of low-dispersion spectra

- Visualization of BAO observations on sky map; Creation of electronic interactive sky map and search system

- Scientific analysis of existing observational material and providing new research possibilities; Proposing and discussing new research projects

BAO PAP webpage (http://www.aras.am/PlateArchive/; Figure 2) is open and contains a lot of information on $\mathrm{BAO}$ observations, previous digitization projects, present Project details, teams, follow-up research projects, deliverables and related links (many items will be filled in during the next months). The main products will be "Data Access" and "Interactive Sky Map". The first one will contain BAO Observational Database, Search by any parameter (Dates / Julian dates, Telescope, Observing modes/methods, Instrument, Receiver, Emulsion, Filters, Seeing, Project name, Project PI, Observers, Targets / coordinates, Sky area, Surface, Scale, Spatial resolution, Spectral range, Spectral resolution, Limiting magnitude, Number of nights, Number of exposures, Links), Data Visualization and Download of the digitized plates, films, part of them or individual objects images or spectra. "Interactive Sky Map" will visualize the observed by BAO telescopes sky and will give possibility to check observed areas for a given observational project, given telescope, observer, observing method, limiting magnitude, etc. There will be possibility to check individual fields for presence and number of plates to propose further research projects. Main expected projects are supposed to be those on variability and proper motions, as well as studies of the Solar System objects.

\section{DFBS and other digitization projects at BAO}

So far, a number of digitization projects have been accomplished at BAO, including the most important one, Digitized First Byurakan Survey (DFBS; http://www.aras.am/Dfbs/dfbs.html; Mickaelian et al. (2007a); Massaro et al. (2008); Mickaelian et al. (2019a)) based on the digitization of the famous Markarian Survey (Markarian et al., 1989).

The Second Byurakan Survey (SBS; Stepanian (2005)) plates are also subject for digitization, as they are hypersensitized and their emulsion is more sensitive for deterioration. 180 plates have been digitized so far. Due to SBS smaller photographic grains, 2400 dpi $(10 \mu \mathrm{m}$ pixel size $)$ is being used and $512 \mathrm{MB}$ files are being obtained for each plate.

Photographic spectra of the FBS blue stellar objects (BSOs) have been obtained using $2.6 \mathrm{~m}$ telescope and UAGS spectrograph on photographic films. 700 such spectra have been scanned with 1600 dpi, 16 bit and $650 \times 21$ pix sizes images were obtained (FBS BSOs; Mickaelian (2008) and late-type 


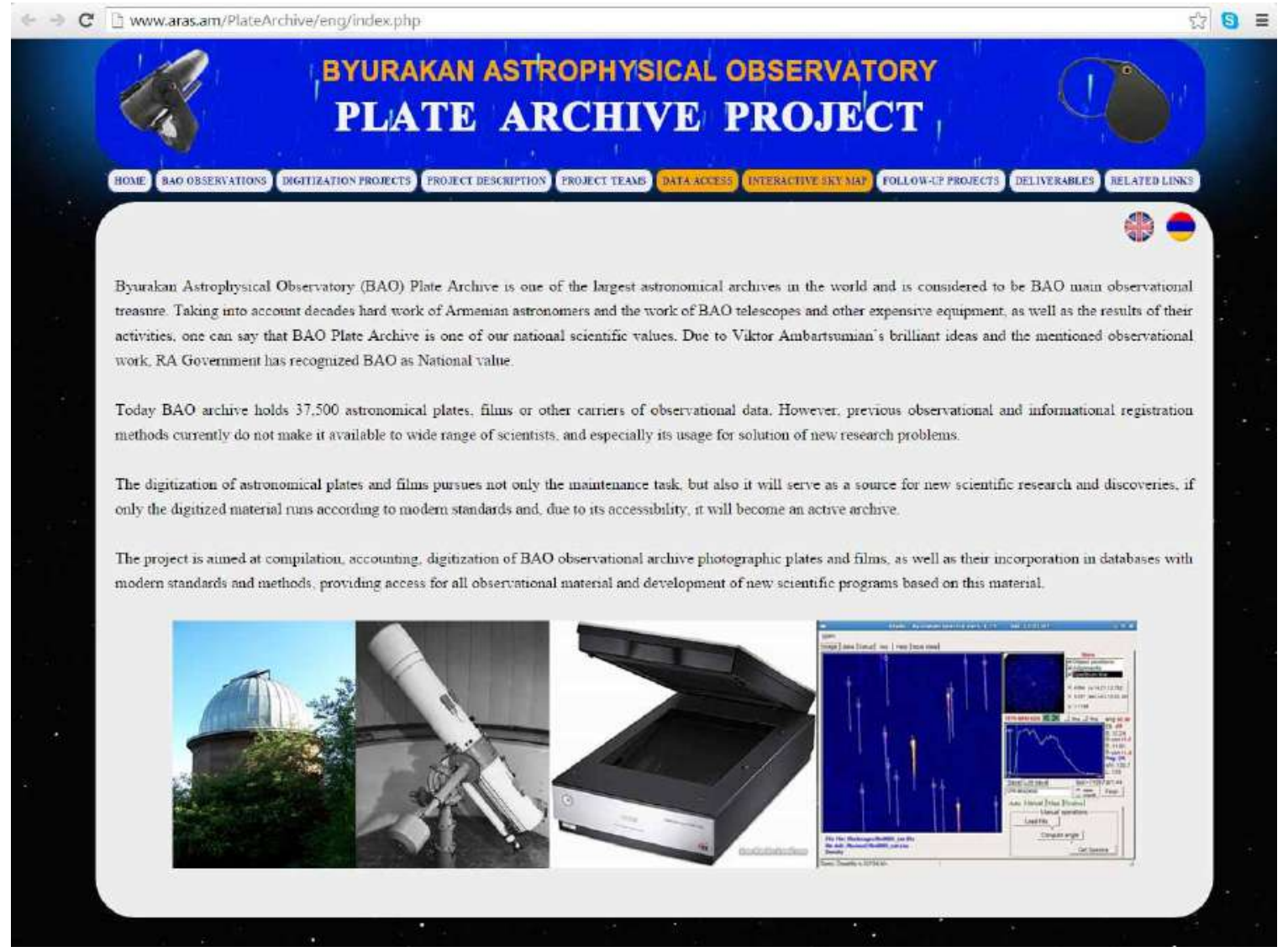

Figure 2. BAO Plate Archive Project webpage.

stars; Gigoyan \& Mickaelian (2012)). All spectra were put in a standard format, so that automatic reduction was possible (Figure 3). 101 FBS blue stellar objects were published and a number of planetary nebulae, white dwarfs, hot subdwarfs and HBB stars have been revealed (Sinamyan \& Mickaelian, 2009).

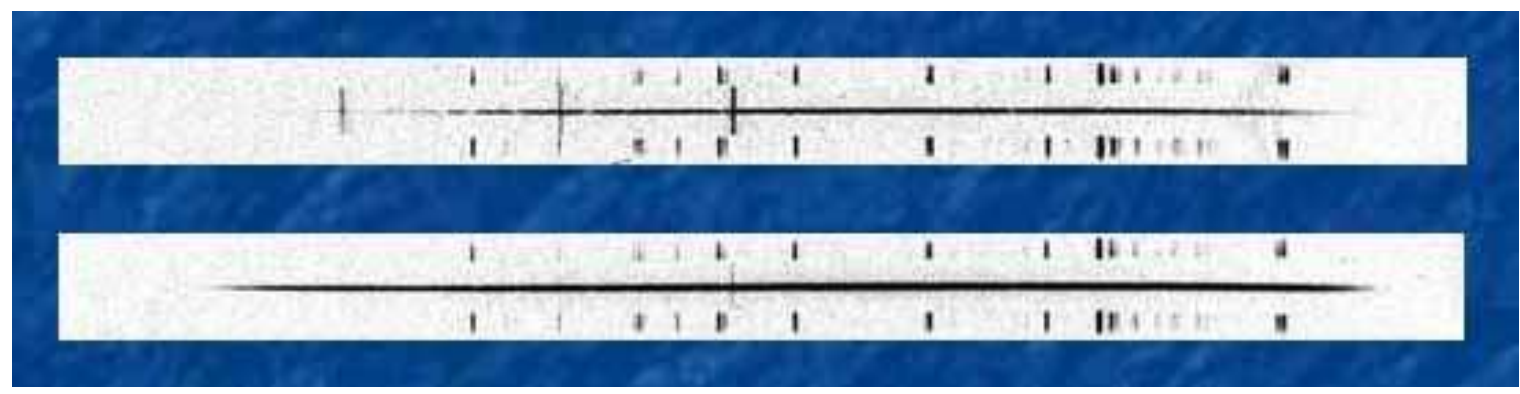

Figure 3. Standard format of FBS spectra with $650 \times 21$ pix sizes images for automatic reduction.

Another project was the study of long-term variability of ON 231, which appeared in the Coma field, where photographic chains for discovery of flare stars were carried out. In total 189 plates with a total number of more than 1200 exposures in 1969-1976 with the Byurakan 21" and 40" Schmidt telescopes were obtained. This was a valuable material for study of ON231 long-term variability (Figure 4; Erastova (2004)).

Having digitized plates and modern digital observational data, a number of efficient research projects have become possible, such as data discovery, spectral analysis, SED building and fitting, modelling, variability studies, cross-matching, etc. Some examples are variability studies (Samus \& Antipin, 2012), Cross-matching of Astronomical Catalogs (Malkov, 2012), Search for Asteroids and Exoplanets using VO tools (Sarkissian et al., 2012).

Our science projects are aimed at discoveries of new interesting objects searching definite types 

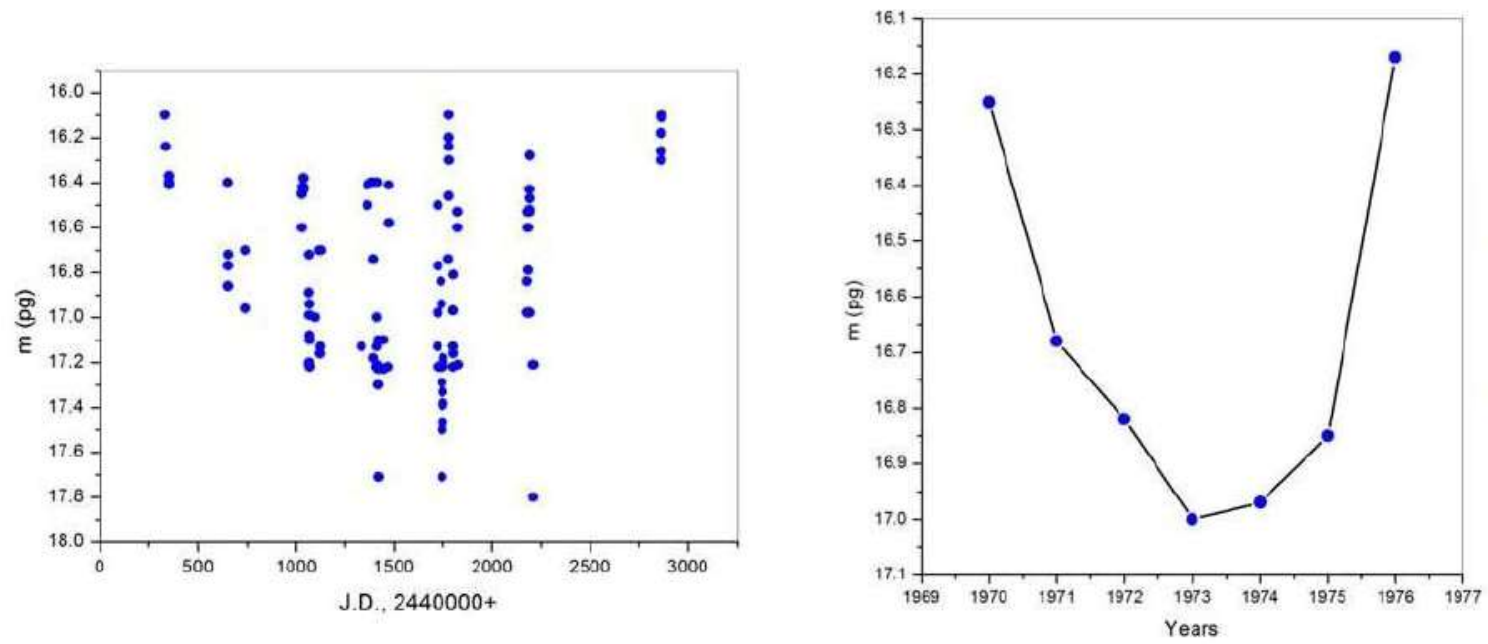

Figure 4. Photometric measurements and variability study of the blazar ON 231 observed in the Coma field.

of low-dispersion spectra in the DFBS, by optical identifications of non-optical sources (X-ray, IR, radio) also using the DFBS and DSS/SDSS, by using cross-correlations of large catalogs and selection of objects by definite criteria, etc.

\section{Summary}

BAO Plate Archive is one of the most important astronomical databases. A project of its digitization and creation of the electronic database is active. At present the main part of the project, the scanning of the plates has produced more than 30,000 digital images of roughly $200 \mathrm{MB}$ files (each image). All they have been stored and double copies are available.

BAO Electronic Database will give access to observing data by various parameters, as shown in Table 2, so that it will fit many requirements for future studies.

Table 2. BAO Electronic Database structure and access modes.

\begin{tabular}{|l|l|}
\hline \multicolumn{2}{|c|}{ BAO Electronic Database } \\
\hline Dates / Julian dates & Targets / coordinates \\
\hline Telescope & Sky area \\
\hline Observing modes/methods & Surface \\
\hline Instrument & Scale \\
\hline Receiver & Spatial resolution \\
\hline Emulsion & Spectral range \\
\hline Filters & Spectral resolution \\
\hline Seeing & Limiting magnitude \\
\hline Project name & Number of nights \\
\hline Project PI & Number of exposures \\
\hline Observers & Links \\
\hline
\end{tabular}

BAO Plate Archive Project will lead to preservation of BAO valuable observational material obtained during 1947-1991. However, our goal is not only to create a passive archive of scanned plates and films, but also to make use of especially those fields, where more studies are possible. Proper motion and variability studies are most important, as time domain material is contained in historical plates. Such possibilities based on DFBS were shown by Mickaelian et al. (2006a); DFBS as a unique database for proper motion, variability studies, and object classification. New variable stars discovered on digitized plates of Moscow collection was reported by Sokolovsky et al. (2014). 
There are a number of further possible research projects that will be conducted having the plates digitized:

- Correction of ephemerides of known asteroids and search for new asteroids (ex. Thuillot et al. (2007); Berthier et al. (2009); Mickaelian et al. (2019b))

- Discovery and study of variable stars (ex. Mickaelian et al. (2011); Nesci et al. (2009))

- Revealing high proper motion stars (ex. Mickaelian \& Sinamyan (2010))

- Study of variability of known blazars and discovery of new blazars

- Revealing Novae and Supernovae progenitors (Nesci et al. (2009))

- Discovery of new QSOs

- Discovery of new white dwarfs (ex. Sinamyan \& Mickaelian (2011))

- Discovery of new late-type stars (ex. Gigoyan et al. (2010, 2019))

- Discovery of optical sources of gamma-ray bursts

- Optical identifications of X-ray, IR and radio sources (ex. Mickaelian \& Sargsyan (2004); Mickaelian \& Gigoyan (2006); Mickaelian et al. (2006b); Hovhannisyan et al. (2009)).

\section{References}

Berthier J., Sarkissian A., Mickaelian A. M., Thuillot W., 2009, ”European Planetary Science Congress 2009, held 14-18 September in Potsdam, Germany, p. 526

Erastova L. K., 2004, A\&AT, 23, 209

Gigoyan K. S., Mickaelian A. M., 2012, MNRAS, 419, 3346

Gigoyan K. S., Sinamyan P. K., Engels D., Mickaelian A. M., 2010, Astrophysics, 53, 123

Gigoyan K. S., Kostandyan G. R., Gigoyan K. K., Zamkotsian F., Sarkissian A., 2019, Astrophysics, 62 , 573

Hovhannisyan L. R., Mickaelian A. M., Weedman D., et al. 2009, AJ, 251, 138

Hudec R., 2014, in Proc. Astroplate-2014 Conf., Eds. L. Mišková \& S. Vítek. Prague, p. 1

Kazantseva L., 2014, in Proc. Astroplate-2014 Conf., Eds. L. Mišková \& S. Vítek. Prague, p. 13

Knyazyan A. V., Astsatryan H. V., Mickaelian A. M., 2016, Astronomical Surveys and Big Data, Eds. A. M. Mickaelian, A. Lawrence and T. Yu. Magakian. ASP Conf. Series, 505, 236

Malkov O. Y., 2012, in Proc. Conf. "50 years of Cosmic Era: Real and Virtual Studies of the Sky". Yerevan, NAS RA, p. 48

Markarian B. E., Lipovetsky V. A., Stepanian J. A., et al. 1989, Comm. SAO, 62, 5

Massaro E., Mickaelian A. M., Nesci R., Weedman D., (Eds.) 2008, The Digitized First Byurakan Survey, ARACNE Editrice, Rome, 78p.

Mickaelian A. M., 2006, Rom. Astron. J., 16S, 23

Mickaelian A. M., 2007, Highlights of Astronomy, 14, 594

Mickaelian A. M., 2008, AJ, 136, 946

Mickaelian A. M., 2012, in Proc. Conf. "50 years of Cosmic Era: Real and Virtual Studies of the Sky". Yerevan, NAS RA. , p. 29 Mickaelian A. M., 2014a, in Proc. Astroplate-2014 Conf., Eds. L. Mišková \& S. Vítek. Prague, p. 109

Mickaelian A. M., 2014b, in Proc. IAU S304: Multiwavelength AGN Surveys and Studies. Vol. 304, p. 1

Mickaelian A. M., Gigoyan K. S., 2006, A\&A, 455, 765

Mickaelian A. M., Sargsyan L. A., 2004, Astrophysics, 47, 213

Mickaelian A. M., Sinamyan P. K., 2010, MNRAS, 407, 681 
Mickaelian A. M., Malkov O. Y., Samus N. N., (Eds.) 2012, Proc. Conf. 50 years of Cosmic Era: Real and Virtual Studies of the Sky. NAS RA, 251 p., Yerevan

Mickaelian A. M., Hagen H.-J., Sargsyan L. A., Mikayelyan G. A., 2005, Catalog No. VI/116 at CDS, Strasbourg

Mickaelian A. M., Nesci R., Cirimele G., et al. 2008, Proc. ESAC Workshop on Astronomical Spectroscopy and Virtual Observatory, Madrid, ESA, p. 29

Mickaelian A. M., Sargsyan L. A., Mikayelyan G. A., 2010, Proc. of Science, 30

Mickaelian A. M., Mikayelyan G. A., Sinamyan P. K., 2011, MNRAS, 415, 1061

Mickaelian A. M., Sargsyan L. A., Mikayelyan G. A., et al. 2006a, Heron Press Sci. Ser., p. 82

Mickaelian A. M., Hovhannisyan L. R., Engels D., et al. 2006b, A\&A, 449, 425

Mickaelian A. M., Gigoyan K. S., Nesci R., Rossi C., 2006c, Mem.S.A.It., 77, 1159

Mickaelian A. M., Nesci R., Rossi C., et al. 2007a, A\&A, 464, 1177

Mickaelian A. M., Sargsyan L. A., Nesci R., et al. 2007b, Highlights of Astronomy, 14

Mickaelian A. M., Astsatryan H. V., Sahakyan V. G., et al. 2009a, Proc. CSIT-2009, p. 420

Mickaelian A. M., Kochiashvili N., Astsatryan H. V., et al. 2009b, Proc. CSIT-2009, p. 424

Mickaelian A. M., Sargsyan L. A., Astsatryan H. V., et al. 2009c, Data Science Journal, 8, 152

Mickaelian A. M., Sargsyan L. A., Gigoyan K. S., et al. 2009d, Rom. Astron. J., 185, 249

Mickaelian A. M., Astsatryan H. V., Knyazyan A. V., et al. 2016a, in Astronomical Surveys and Big Data. Eds. A. M. Mickaelian, A. Lawrence \& T. Yu. Magakian. ASP Conf. Ser., V. 505, p. 16

Mickaelian A. M., Abrahamyan H. V., Andreasyan H. R., et al. 2016b, in Astronomical Surveys and Big Data. Eds. A. M. Mickaelian, A. Lawrence \& T. Yu. Magakian. ASP Conf. Ser., V. 505, p. 262

Mickaelian A. M., Sargsyan L. A., Mikayelyan G. A., Erastova L. K., Gigoyan K. S., Sinamyan P. K., Hovhannisyan L. R., 2019a, ASP Conf. Ser., V. 520, p. 105

Mickaelian A. M., Sarkissian A., Berthier J., Meftah M., Thuillot W., Vachier F., 2019b, Icarus, 330, 5

Nesci R., Mickaelian A. M., Rossi C., 2009, ATel, 2338, 1

Nesci R., Bagaglia M., Nucciarelli G., 2014a, in Proc. Astroplate-2014 Conf., Eds. L. Mišková \& S. Vítek. Prague, p. 75

Nesci R., Gaudenzi S., Rossi C., Pezzotti C., Gigoyan K., Mauron N., 2014b, in Proc. Astroplate-2014 Conf., Eds. L. Mišková \& S. Vítek. Prague, p. 91

Osborn W., 2014, in Proc. Astroplate-2014 Conf., Eds. L. Mišková \& S. Vítek. Prague, p. 15

Samus N. N., Antipin S., 2012, in Proc. Conf. "50 years of Cosmic Era: Real and Virtual Studies of the Sky". Yerevan, NAS RA, p. 39

Sarkissian A., Arzoumanian E., Mickaelian A. M., et al. 2012, in Proc. Conf. "50 years of Cosmic Era: Real and Virtual Studies of the Sky". Yerevan, NAS RA, p. 56

Sinamyan P. K., Mickaelian A. M., 2009, Astrophysics, 52, 76

Sinamyan P. K., Mickaelian A. M., 2011, Astrophysics, 54, 403

Sokolovsky K. V., Antipin S. V., Zubareva A. M., et al. 2014, ARep, 58, 319

Stepanian J. A., 2005, RMxAA, 41, 155

Stupka J., Benesova E., 2014, in Proc. Astroplate-2014 Conf., Eds. L. Mišková \& S. Vítek. Prague, p. 31

Thuillot W., Berthier J., Sarkissian A., et al. 2007, Highlights of Astronomy, 14, 616

Tsvetkov M., Tsvetkova K., 2012, in Proc. IAU S285: New Horizons in Time-Domain Astronomy. Cambridge Univ. Press, p. 417

Véron-Cetty M.-P., Véron P., 2010, A\&A, 518, 10 